\title{
Configural integration of temporal and contextual information in rats: Automated measurement in appetitive and aversive preparations
}

\author{
Natasha M. Dumigan • Tzu-Ching E. Lin • Mark Good • \\ Robert C. Honey
}

Published online: 12 March 2015

(C) Psychonomic Society, Inc. 2015

\begin{abstract}
Two experiments investigated the capacity of rats to learn configural discriminations requiring integration of contextual (where) with temporal (when) information. In Experiment 1, during morning training sessions, food was delivered in context $\mathrm{A}$ and not in context $\mathrm{B}$, whereas during afternoon sessions food was delivered in context $\mathrm{B}$ and not in context A. Rats acquired this discrimination over the course of 20 days. Experiment 2 employed a directly analogous aversive conditioning procedure in which footshock served in place of food. This procedure allowed the acquisition of the discrimination to be assessed through changes in activity to the contextual+temporal configurations (i.e., inactivity or freezing) and modulation of the immediate impact of footshock presentations (i.e., post-shock activity bursts). Both measures provided evidence of configural learning over the course of 12 days, with a final test showing that the presentation of footshock resulted in more post-shock activity in the nonreinforced than reinforced configurations. These behavioral effects reveal important parallels between (i) configural discrimination learning involving components allied to episodic memory and (ii) simple conditioning.
\end{abstract}

Keywords Acquisition · Associative learning - Configural learning $\cdot$ Context

N. M. Dumigan • T.-C. E. Lin • M. Good • R. C. Honey

Cardiff University, Cardiff, UK

R. C. Honey $(\bowtie)$

School of Psychology, Cardiff University, Park Place, Cardiff CF10

3AT, UK

e-mail: honey@cardiff.ac.uk
An animal will often be better placed to survive if its learning about target stimuli is contextualized with other, non-target information: the deep purple berries on one variety of shrub might be nutritious while those on another might be poisonous, and drinking from a specific watering hole might be safe at daybreak but dangerous at dusk. One can demonstrate such integration through the ability of rats to acquire configural discriminations involving contexts. For example, rats can learn that when they are placed in one context (A; e.g., a chamber decorated with spotted wallpaper) presentations of a tone (X) are followed by food and those of a clicker (Y) are nonreinforced, whereas when they are placed in a different context (B; e.g., a chamber decorated with checked wallpaper) presentations of $\mathrm{Y}$ are reinforced and those of $\mathrm{X}$ are not (e.g., Honey \& Watt, 1999; Preston et al., 1986).

Most often configural discriminations involve stimuli that are present in an animal's immediate (external) environment: the vegetation and the position of the sun in the sky, to return to our opening examples. However, an animal who can only learn about such configurations is unlikely to survive as long as one who can make use of information that is (i) temporally or spatially remote from stimuli that are currently impinging on them (e.g., when fallen berries have scattered some distance from where they originated), or (ii) internally generated (e.g., when cloud cover means that there are no obvious visual cues about time of day). There is evidence that rats can form integrated configural memories involving stimulus traces (see Lin \& Honey, 2010), and some evidence that is consistent with the possibility that rodents can learn configural discriminations involving time of day.

Rats can learn to choose the left choice arm in a T-maze rather than the right arm to gain food in the morning, and to do the reverse to gain food in the afternoon (e.g., Means et al,. 2000a; see also, Means et al,. 2000b; Thorpe et al,. 2003). However, such observations simply require the cues that are correlated with different times of day to become linked to 
specific responses (e.g., turn left or right). Evidence that such cues become configured with information from other sources comes from studies that have investigated whether rats can learn what happened where and when (Iordanova et al,. 2008; see also, Iordanova, Burnett, Good \& Honey, 2011a; Iordanova et al,. 2009; Iordanova, Good \& Honey, 2011b; for a review, see Honey et al,. 2014). In these studies, in the morning, placement in context $\mathrm{A}$ is associated with presentations of one auditory stimulus $(\mathrm{X})$ and placement in context $\mathrm{B}$ is associated with a second auditory stimulus (Y); and in the afternoon the contexts in which the auditory stimuli are presented are transposed. The fact that these configurations have been encoded can be revealed by showing that pairing $\mathrm{X}$ with shock (at midday in a third context) results in more fear in the time-of-day+ context configurations in which $\mathrm{X}$ had been presented (morning $+\mathrm{A}$ and afternoon $+\mathrm{B}$ ) than in the other configurations (morning $+\mathrm{B}$ and afternoon $+\mathrm{A}$; see also, Cain et al,. 2004; but see, McDonald et al,. 2002).

Here, we sought rather more direct evidence that temporal information can be integrated with contextual information using standard configural training procedures (cf. Means et al., 2000ab) that allowed both an automated and relatively continuous measurement of the process of integration (cf. Iordanova et al., 2008). Assays that employ automated measures are useful insofar as they obviate the needs for timeconsuming video scoring or the experimenter being in the room when the rat is being tested; and continuous measures allow assessment of the rate at which integrated configural memories are formed in rats (cf. Clayton \& Dickinson, 1998). To do this, we report two experiments that investigated the capacity of rats to acquire configural discriminations in which critical components of the configurations were cues associated with the times of day (morning or afternoon) when animals were placed in two contexts, A and B. Experiment 1 employed an appetitive reinforcer (food) and Experiment 2 an aversive reinforcer (footshock). Each day, rats were placed in two contexts (A and B) in the morning and the same two contexts in the afternoon. In the morning, food (or shock) was presented in context A (but not in B) and in the afternoon food (or shock) was presented in context B (but not in A). The development of the appetitive configural discrimination (in Experiment 1) was assessed by the tendency of the rats to enter the food well when presented with the four configurations, and that of the aversive configural discrimination (Experiment 2) by the tendency of rats to suppress ongoing behavior when presented with the same configurations.

Importantly, Experiment 2 also allowed an assessment of whether or not configural learning involving where and when footshock is delivered has the same behavioral sequelae as more standard forms of Pavlovian conditioning: where the immediate unconditioned response to shock (an activity burst; e.g., Fanselow, 1982) contrasts with the conditioned response (freezing or inactivity), and the unconditioned response is modulated by the presence of an effective conditioned stimulus (cf. Wagner, 1981). Briefly, Wagner's (1981) model assumes that the formation of an association between the memory of a conditioned stimulus and an unconditioned stimulus allows the future presentation of the conditioned stimulus to place the unconditioned stimulus into a refractory state in which it is less likely to provoke its unconditioned response (e.g., an activity burst). We assessed such modulation by presenting shock in where + when configurations in which shock had either been delivered or not. A so-called conditioned diminution of the unconditioned response would be evident if the post-shock activity burst was less marked in the configuration in which shock had been delivered than in the configuration in which no shock had been delivered.

\section{Experiments 1 and 2}

In Experiment 1, rats were placed in two contexts (A and B; a spotted and a checked chamber) in both the morning and afternoon. During the morning sessions, food pellets were delivered in $\mathrm{A}$ but not $\mathrm{B}$, whereas in the afternoon sessions, food pellets were delivered in B but not A. Acquisition of this appetitive discrimination was assessed by recording the tendency of rats to approach the food well during the food-free periods at the outset of each of the four types of trial. Experiment 2 used the same design but footshock was delivered in the morning in context $\mathrm{A}$ but not $\mathrm{B}$, and in the afternoon footshock was delivered in context $\mathrm{B}$ but not $\mathrm{A}$. Acquisition of this aversive discrimination was measured using an automated system that recorded the levels of general activity in footshock-free periods at the start of each of the sessions (cf. Lin et al,. 2013). It was anticipated that the level of activity would be lower in the configurations paired with foothshock (i.e., morning $+\mathrm{A}$ and afternoon $+\mathrm{B}$ ) than those that were not (i.e., morning $+\mathrm{B}$ and afternoon $+\mathrm{A}$ ). Using the same system, the levels of post-shock activity were measured. It is well established that the delivery of footshock can result in a post-shock activity burst (e.g., Fanselow, 1982) and we assessed whether this burst changed over the course of training. Finally, we presented footshock in previously reinforced and nonreinforced configurations to assess whether the response to shock was modulated by whether the configuration had been paired with shock or not (cf. Wagner, 1981; see also, for example, Honey, Good \& Manser, 1998a; Honey, Watt \& Good, 1998b; Honey \& Good, 2000).

\section{Method}

Subjects Sixteen naïve Lister hooded rats (Rattus norvegicus; supplied by Harlan Olac Ltd, UK) were used in each experiment. The rats in Experiment 1 were $\approx 3$ months old at the start 
of the experiment (mean ad libitum weight $=298 \mathrm{~g}$; range: 278 - $314 \mathrm{~g}$ ) as were those in Experiment 2 (mean ad libitum weight $=287 \mathrm{~g}$; range $275-311 \mathrm{~g}$ ). They were maintained at $85 \%$ of their ad libitum weights by giving them a restricted amount of food at the end of the day ( $\approx 1830 \mathrm{hrs})$, and housed in pairs in a colony room that was illuminated between the hours of 8 a.m. and 8 p.m. Rats were weighed on each day and separated for feeding if this was required to maintain their weights. Behavioral training began at, approximately, 09:30 each day.

Apparatus Experiment 1 employed a set of four chambers $(23.0 \mathrm{~cm} \times 24.5 \mathrm{~cm} \times 21.0 \mathrm{~cm}, \mathrm{~L} \times \mathrm{W} \times \mathrm{H}$; supplied by Camden Instruments Ltd., United Kingdom) arranged in a $2 \times 2$ array. The chambers were constructed from three aluminum walls, an aluminum ceiling, and a plastic wall that served as the door to the chamber. The ceilings and walls of the top pair of boxes in the array were decorated with spotted laminated paper (black circles on a white background), whereas the walls and ceiling of the lower two chambers were decorated with black and white checked laminated paper (for further details, see Honey \& Watt, 1999). There was a food well in the left hand aluminium wall into which $45 \mathrm{mg}$ of food pellets (supplied by P. J. Noyes, Lancaster, NH) could be delivered. A top-hinged transparent plastic flap guarded access to this food well. Food-well entries were automatically recorded when the top-hinged magazine flap was pushed approximately $3 \mathrm{~mm}$. A series of stainless steel rods, $0.50 \mathrm{~cm}$ in diameter and $1.5 \mathrm{~cm}$ apart (centre-to-centre), served as the chamber floor, below which was a tray containing a $24 \mathrm{~cm} \times$ $23 \mathrm{~cm}$ absorbent sheet. The chamber received local illumination from a single $15-\mathrm{V}, 24-\mathrm{W}$ jewel light positioned in the centre of the ceiling, and ambient illumination from the striplight in the experimental room. The doors of the soundattenuating shells in which the chambers were housed were left open throughout training.

Experiment 2 used a set of eight operant chambers $(30.5 \mathrm{~cm} \times 26 \mathrm{~cm} \times 20 \mathrm{~cm}$; Test chamber 80004 -D001; supplied by Campden Instruments Ltd., Loughborough, England) arranged in a $4 \times 2$ array that were positioned within sound-attenuating boxes. Each chamber had two aluminum sidewalls, a transparent Perspex back wall and a transparent Perspex ceiling. The front walls were also constructed from transparent Perspex and served as the doors to the chamber. The walls of the upper row of boxes were decorated with spotted wallpaper and those of the lower row were decorated with checked wallpaper. The chambers were illuminated by a 3-W light bulb, with a white plastic cover, positioned centrally and at $13.5 \mathrm{~cm}$ above the floor of the left aluminium wall. There was a stainless steel grid floor, constructed from 19 bars (diameter $0.47 \mathrm{~cm}$, spacing from bar centre to bar centre $1.07 \mathrm{~cm}$ ) to which a $0.5-\mathrm{s} .0 .64 \mathrm{~mA}$ electric shock could be delivered using a scrambled shocker (Campden Instruments
Ltd. Model HSCK1000). Beneath the floor was a tray that was lined with absorbent paper. The level of rat activity in the chambers was measured using an ambulatory monitor (Campden Instruments Ltd. Model 80004 AM) that consisted of a horizontal strip attached to the back wall of the chamber and another to the front wall positioned $3.0 \mathrm{~cm}$ above the grid floor. These strips contained three infrared light sources and photo beam detectors that were located $3.0 \mathrm{~cm}$ from the left hand wall, in the centre of the chamber, and $3.0 \mathrm{~cm}$ from the right hand wall. Detection of the presence of the rat in the area covered by a photo beam followed by detection of the absence of the rat in this area, as the rat moved, was recorded as a value of 1 . The number of times this occurred for each of the three beams gave a single value for the total movement made by the rat. A computer (Mark II Control Unit) controlled the apparatus, operated the programs (using Behavioural Net Controller Control 1.0), and recorded ambulatory movement data (all equipment was supplied by Campden Instruments Ltd.).

\section{Procedure}

Experiment 1: Appetitive configuration discrimination On the first day, in two 10-min sessions, conducted between 1:30 and $3: 30$, rats were trained to retrieve food pellets from a food well in undecorated chambers. In the first session, the flaps in front of the food wells were fixed in a raised position to allow rats ready access to the food pellets; and in the second session these flaps were lowered and rats had to move them to gain access to the food pellets. During both sessions, 20 food pellets were delivered two at a time on a variable time 60 -s schedule. Successful food well training was followed by 20 days of discrimination training. On each day, rats were placed in the two contexts (spotted and checked) in the morning and the same two visual contexts in the same sequence in the afternoon (spotted and then checked for half of the rats, and checked and then spotted for the remainder). For a given training day, the order in which the contexts were presented for a given rat was consistent, but across days the order was pseudo-randomised with the constraint that there were no more than two consecutive days with the same order. Morning sessions took place between 09:30 and 11:30, and afternoon sessions took place between 4:30 and 6:30. Each context placement lasted $5 \mathrm{~min}$, and there was a $5 \mathrm{~min}$ interval between the two morning and afternoon sessions during which rats were placed in a holding cage outside the testing room. In the morning, during placement in one context (e.g., spotted) two food pellets were delivered to the food well every 30 s (resulting in a total of 20 pellets delivered per session), and during placement in the other context (e.g., checked) no food pellets were delivered. In the afternoon this arrangement was reversed. Which of the contexts was reinforced in the morning or the afternoon was fully counterbalanced. 
Following the end of each pair of sessions the rats were taken back to the colony room and replaced in their holding cages.

To reduce the likelihood of rats learning that food would not be delivered for the first 30-s of any session, each cycle of 4 training days had the following structure. On the first two days of the cycle, on placement in the "reinforced" contexts, an additional 2 pellets were present in the food well. For the following two days of the cycle, no food pellets were present in the food well for the first 30 -sec periods. The number of food well entries made by a rat during this first 30 s reinforcerfree periods during the second pair of training days in each cycle was used to assess the acquisition of the discrimination. To do so, a discrimination ratio was used: food well entries per minute during reinforced sessions (e.g. spotted + morning) divided by the combined number of entries per minute during reinforced and non-reinforced sessions (e.g. spotted +morning, and checked+morning), for each morning and afternoon session. A score of 0.50 indicates that the number of food well entries was the same during the reinforced and nonreinforced sessions, whereas scores greater than 0.50 indicate that responding was greater during reinforced than nonreinforced sessions. The raw rates of responding on the reinforced and nonreinforced trials were also reported.

Experiment 2: Aversive configuration discrimination To acclimate rats to the procedure, on both of the first two days each rat was placed into an undecorated operant chamber for $\approx$ $20 \mathrm{~min}$. The lights in the testing room were turned off and the house light in each chamber was illuminated for 20-min once each squad of 8 rats had been placed in the chambers. These sessions, conducted between 12 p.m. and 2 p.m., were followed by 12 days of training. On each day, rats were placed in the two contexts (spotted and checked) in the morning and afternoon in the same sequence (spotted and then checked for half of the rats, and checked and then spotted for the remainder). As in Experiment 1, across days the sequence in which rats were placed in the two contexts varied according to a pseudo-randomised sequence. Each exposure to a context lasted for $3 \mathrm{~min}$ and there was an interval of $5 \mathrm{~min}$ between the pairs of exposures (to the spotted and checked contexts) in the morning and afternoon. In the morning, during exposure to one of the contexts (e.g. spotted) two mild electric shocks were delivered through the grid floor of the chamber, one after the first minute and another after the second minute of the 3minute session. During exposure to the other visual context (e.g. checked) no shocks were delivered. In the afternoon this arrangement was reversed (e.g. shocks were delivered in the checked but not in the spotted context). Note that in Experiment 2 there were no sessions on which shock was presented immediately after the rats entered the chamber because such presentations disrupt fear conditioning (Fanselow, 1986). Details of the procedure that have not been mentioned were the same as for Experiment 1.
To assess acquisition of the configural discriminations during training, a discrimination ratio was used: activity during first 30s of session without shocks (e.g., checked+morning) divided by the combined activity during the first 30 s of both sessions at that time of day (e.g., spotted+morning, and checked+morning). Using this measure, a score of 0.50 indicates that rats were equally active in the context in which shock is delivered and the context where no shocks were delivered, and scores above 0.50 indicate rats are more active in sessions without shocks than with shocks. As in Experiment 1 , the raw rates of responding on the reinforced and nonreinforced trials were also reported. In addition, we assessed the impact of the shocks themselves by measuring activity in the 30-s period that immediately followed shock presentation, and during the same time periods in the corresponding sessions where no shocks were delivered.

On the thirteenth day, rats were again placed in the two contexts at both times of day, but in every session two shocks were delivered to the grid floor (one after $1 \mathrm{~min}$ and the other after $2 \mathrm{~min}$ ). Activity was measured during the 30s immediately following each shock presentation. Our analysis focused on the impact of the first presentations of shock in the two morning sessions, because the rats became inactive in both contexts in the afternoon.

\section{Results and discussion}

Experiment 1: Appetitive configuration discrimination The upper panel of Fig. 1 depicts the mean of the morning and afternoon discrimination ratios for the ten days of training on which food was not presented in the first 30 -sec periods (i.e., days $3,4,7,8,11,12,15,16,19,20)$. Inspection of this figure indicates that the discrimination ratios increased across training. ANOVA confirmed that there was a main effect of day $(F(9,135)=2.10, p<0.05)$, and subsequent one-sample $t$ tests revealed that the discrimination ratios were significantly different from 0.50 on days $8,16,19$ and 20 (smallest $t(15)=$ $2.21, p<0.05$ ). The lower panel depicts the rates of responding on reinforced and nonreinforced trials that were used to calculate the ratios. It is evident that there was a marked difference in the rates of responding on the reinforced and nonreinforced trials by the final stages of training. ANOVA revealed a main effect of day, $F(9,135)=4.02, p<0.01$, a main effect of trial type, $F(1,15)=10.88, p<0.05$, and no interaction between these factors, $F(9,135)=1.85, p=.065$. There was a significant difference between the rates of responding on reinforced and nonreinforced trials on days $8,15,16,19$ and 20 (smallest $t(15)=2.39, p<0.05$ ). These results demonstrate that rats can learn an appetitive configural discrimination where the configuration of the context (spotted or checked) and time of day (morning or afternoon) indicates whether or not food will be delivered. 

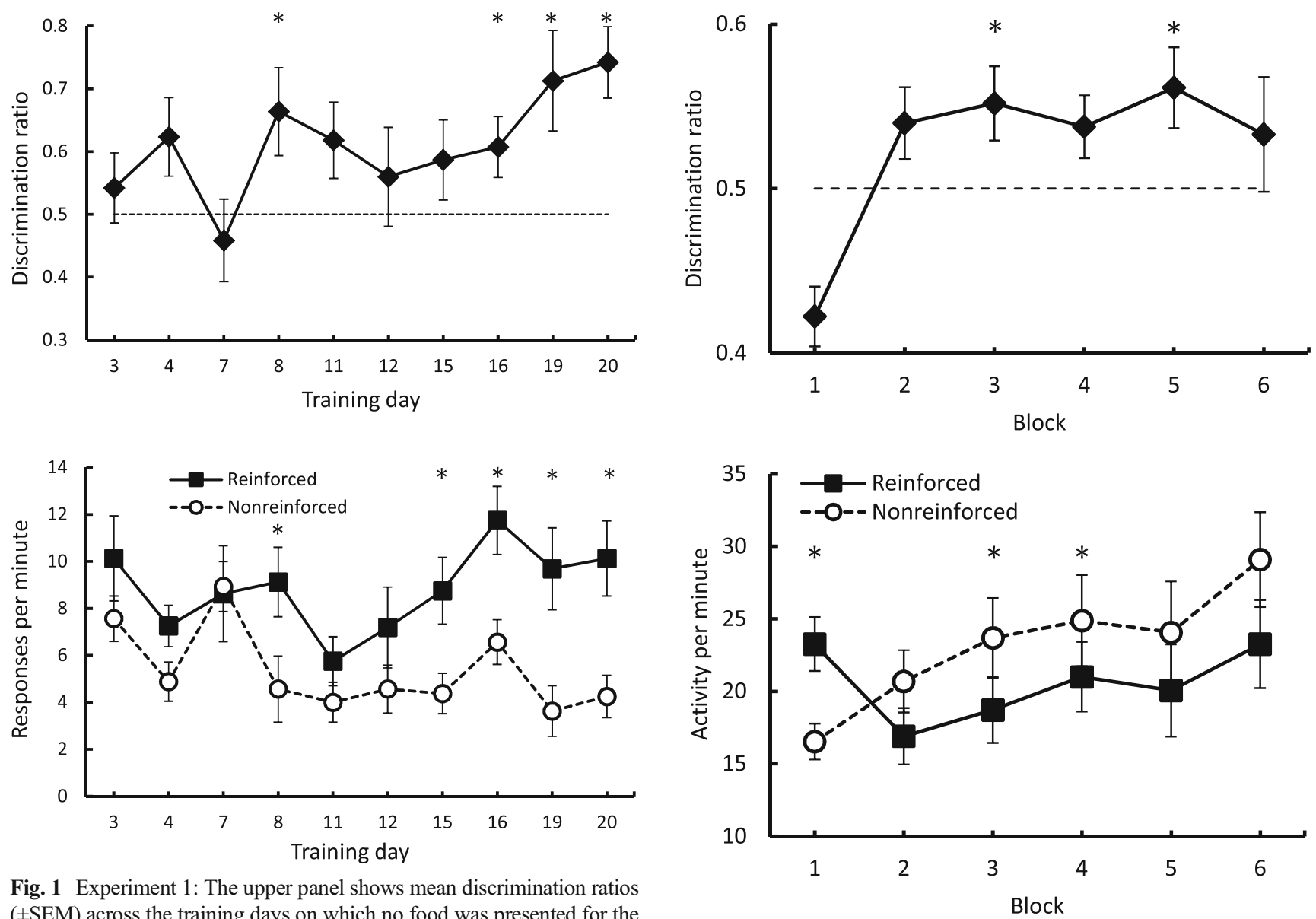

Fig. 1 Experiment 1: The upper panel shows mean discrimination ratios $( \pm$ SEM) across the training days on which no food was presented for the first $30 \mathrm{~s}$ in any of the configurations (i.e., days 3, 4, 7, 8, 11, 12, 15, 16, 19, and 20). These ratios are derived from the food-free 30 -sec periods at the start of the sessions. The lower panel shows the mean rates of responding $( \pm \mathrm{SEM})$ on reinforced and nonreinforced trials that were used to calculate the ratios

Experiment 2: Aversive configuration discrimination The upper panel of Fig. 2 shows the discrimination ratios for the six, two-session blocks of training. ANOVA revealed a significant main effect of block $(F(5,75)=4.04, p<.005)$, with the discrimination ratios being significantly higher than 0.50 on blocks 3 and 5 (smallest $t(15)=2.29, p<.05$ ). The fact that the discrimination ratios are below 0.50 on the first block $(t(15)=4.27, p<0.005$, suggests that the recent presentation of shock reduces activity in an immediately succeeding session, or the recent presentation of no shock increases activity in a succeeding session. These effects of trial sequencing counteract what would be expected on the basis of rats learning the configural discrimination. The lower panel depicts the rates of responding on reinforced and nonreinforced trials that were used to calculate the ratios. It is evident over the course of training there was a gradual increase in activity, perhaps reflecting the conditioned modulation in responding to footshock (see below), and that after the first block of training there was less activity on the reinforced than the nonreinforced trials. ANOVA revealed no significant effect of block,

Fig. 2 Experiment 2: Mean discrimination ratios $( \pm$ SEM) across the 6 blocks of training. These ratios are derived from the footshock-free 30 -sec periods at the start of the sessions. The lower panel shows the mean rates of activity ( \pm SEM) during the reinforced and nonreinforced trials that were used to calculate the ratios

$F(5,75)=2.14, p=0.07$, a main effect of trial type, $F(1,15)=$ $12.04, p<.005$, and an interaction between these factors, $F(5,75)=4.49, p<.005$. There were differences in responding between the trial types on blocks 1,3 and 4 (smallest $t(15)=3.21, p<.05$ ). These results complement those from Experiment 1 in confirming that rats can learn a configural discrimination where the configuration of the context (spotted or checked) and time of day (morning or afternoon) indicates whether or not shock will be delivered.

Post-shock activity during training The left panel of Fig. 3 shows the levels of post-shock activity in the 30 -sec periods after the presentation of the two footshocks in each of the six, two-session blocks of training; and the activity levels in the corresponding periods when shock was not delivered. Inspection of this figure suggests that there was a general increase in activity across training, and that the presence of shocks increased activity in the post-shock period (relative to the equivalent period in the context + time of day configurations 


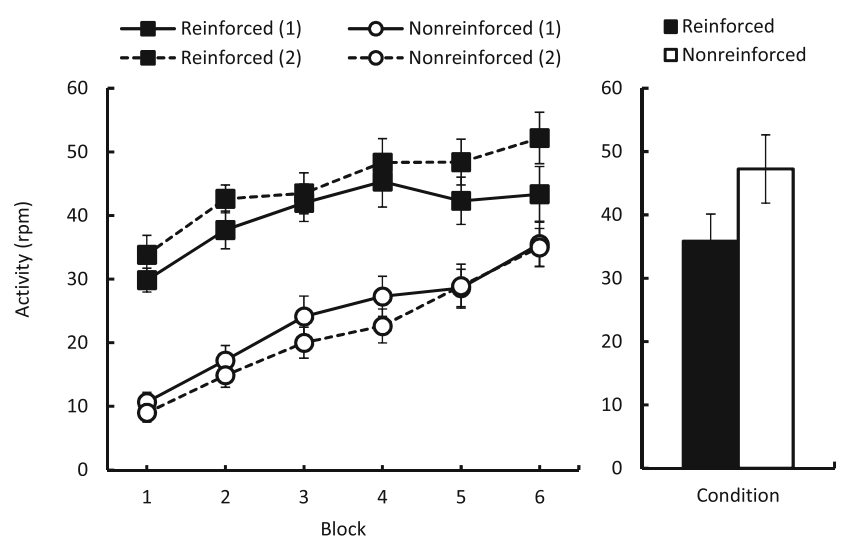

Fig. 3 Experiment 2: Mean rates of activity (in responses per minute; \pm SEM) during the two periods (1 and 2) that immediately followed the two footshocks on reinforced trials, and during equivalent periods ( 1 and 2) on nonreinforced trials (left panel); and mean rates of post-shock activity during a morning test where footshocks were presented in the previously reinforced and nonreinforced configurations (right panel)

where no shock was present). There was also some indication that the second shock increased activity more than the first shock, and that in the nonreinforced configurations there was a reduction in activity between the corresponding first and second periods within a session. These impressions were confirmed by an ANOVA, which revealed a significant main effect of shock $(F(1,15)=151.49, p<.001)$, no effect of shock number $(F(1,15)=1.96, p>.05)$, and an effect of block $(F(5,75)=17.31, p<.001)$. There was an interaction between shock and shock number $(F(1,15)=22.04$, $p<.001)$, an interaction between shock and block $(F(5$, $75)=4.55, p<.005)$, no interaction between block and shock number, and no three-way interaction (largest $F(5,75)=1.43$, $p>.05)$. A secondary ANOVA on the reinforced trials revealed an effect of shock number $(F(1,15)=11.12, p<.01)$, an effect of block $(F(5,75)=8.08, p<.001)$, and no interaction between these factors $(F<1)$. A parallel ANOVA for the nonreinforced trials revealed an effect of block $(F(5,75)=$ $20.83, p<.001)$, period within session $(F(1,15)=6.43$, $p<.05)$, and no interaction $(F(5,75)=1.41, p>.05)$.

Post-shock activity during the test The mean levels of activity during the 30s following the first footshock in each of the morning sessions of the test day are shown in the right panel of Fig. 3. The levels of activity were significantly greater when the footshock was presented in the previously nonreinforced context + time day configuration than in the reinforced configuration, $t(15)=2.22, p<0.05$. These results, involving the modulation of the unconditioned response provoked by shock, provide a second assay for the acquisition of the configural discrimination, and suggest one explanation for the loss of this discrimination in the later blocks of training: a reduction in the effectiveness of shock as training progressed.

\section{General discussion}

Two behavioral procedures are described that provide automated measurements of the acquisition of configural discrimination involving time of day and contextual cues. One procedure used an appetitive reinforcer and approach of the food well as the conditioned response in conventional operant chambers; and the second procedure employed an aversive reinforcer and activity as the conditioned response in chambers equipped with an ambulatory monitor. Both procedures produced robust changes in behavior (cf. Means et al. 2000ab; Thorpe et al., 2003) similar to those seen when rats are given discriminations where the configurations of contextual with auditory cues signalled whether or not food would be delivered (e.g., Honey \& Watt, 1999). There are two specific aspects of the results that are worth dwelling on: The difficulty of the two configural discriminations and the nature of the cues correlated with times of day that the rats might have used.

An animal that can rapidly form episodic-like (whatwhere-when) memories (cf. Clayton \& Dickinson, 1998) should be well placed to acquire configural discriminations that require them to learn where and when food or shock are delivered. However, the appetitive and aversive discriminations in Experiments 1 and 2 took many days to emerge. This observation is consistent with results reported by Iordanova et al. (2008, 2009, 2011ab), in which rats were exposed to four configurations (morning + context $\mathrm{A}+$ tone, morning + contextB + clicker, afternoon + context $\mathrm{A}+$ clicker, afternoon + contextB + tone) on each of four days before the critical test. That is, in neither type of procedure was there evidence that the configural memories developed particularly rapidly (cf. Honey \& Watt, 1999). One could argue that our procedures were not sensitive to capturing the rapid acquisition of configural memories. This argument seems reasonable in the context of Experiment 1, but the procedure used in Experiment 2 was sensitive to observing some differences early in training. The most economical interpretation of these differences was that they reflected the fact that early in training the presentation of shock (or no shock) in the first of a pair of sessions (at a given time of day) directly affected performance in the second session (at that time of day). However, the observation that rats can learn configural discriminations involving cues associated with different times of day raises the issue of which cues provide a basis for this capacity (see Means et al,. 2000a). We have supplementary analyses that bear on some of them.

Supplementary analyses The facts that the procedures were conducted in a quiet experimental room, within a quiet laboratory, with weekly (not daily) changes to bedding means that it is unlikely that there were auditory cues that distinguished between morning and afternoon sessions. However, a related possibility is that there were odor cues that distinguished 
between these sessions. For example, for the first two squads of rats in the morning the chambers might have a different odour than when they re-enter the same chambers in the afternoon, after other squads of rats have been in the chambers. This form of account seems less plausible for later squads of rats. For these rats the odors in the chambers in the afternoon are likely to be similar to those present in the morning, having been previously occupied by the same squads of rats in both sessions. In any case, the prediction that the discrimination ratios for rats tested earlier in the day should be better than those tested later in the day was not borne out in the data. For the final four test days of Experiment $1(15,16,19,20)$ the discrimination ratios for the first two $(n=8)$ and last two squads $(n=8)$ were 0.66 and 0.66 , respectively; and for the final three blocks of training in Experiment 2 the corresponding scores were: Squads 1 and $2(n=8)=0.55$, and Squads 3 and $4(n=8)=0.54$. ANOVA with squad and experiment as factors revealed an effect of experiment $(F(1,28)=9.00$, $p<.01)$, no effect of squad $(F<1)$ and no interaction between the two factors $(F<1)$. These four discrimination ratios were each different from 0.50 (smallest $t(7)=2.43, p<.05$ ), and they provide little support for the contention that differences in odors (generated by the rats) between the morning and afternoon were used to discriminate between the different times of day. A different possibility is that rats were using an alternation strategy. For example, they might have learned that if food (or shock) was presented in the spotted box on the immediately preceding trial then it would be presented in the checked box on the next trial. If this was the case, then one might predict that discriminative performance would be superior when the immediately preceding reinforced trial occurred within a day (i.e., in the morning) than across days (i.e., in the afternoon of the previous day). However, in Experiment 1 a paired $t$-test conducted on the pooled discrimination scores from the final 4 test days $(15,16$, 19 , and 20) revealed no significant difference between the morning sessions (.67), when the preceding reinforced trial was on the previous afternoon, and afternoon sessions (.66), for which the preceding reinforced trial was in the morning $(t(15)=0.16, p>.87)$. Similarly, in Experiment 2 a paired t-test conducted on the pooled scores for the final 3 blocks of training revealed no significant difference between the discrimination ratios in the morning (.53) and afternoon (.55) sessions $(t(15)=0.77, p>.45)$.

There are other potential mediators of discriminative performance in Experiments 1 and 2. Thus morning and afternoon sessions might be correlated with differences in either the rats' state of food deprivation or arousal, and they might encode cues correlated with these differences. In keeping with this possibility, in Experiment 1 the overall levels of magazine entries during the reinforcer-free periods at the start of the sessions was higher in the afternoon $(7.9 \mathrm{rpm})$ than in the morning $(6.2 \mathrm{rpm} ; F(1,15)=15.48, p<.05)$; and this difference was also evident during the final four test sessions $(15,16,19$, 20 ) when the discrimination was most evident (afternoon= $8.4 \mathrm{rpm}$ and morning $=6.4 \mathrm{rpm} ; F(1,15)=5.07, p<.05)$. However, time of day ratios (rate of food well entries in the afternoon divided by the rate of entries at both times of day) did not correlate with configural discrimination ratios when both were pooled across the entire course of training $(r=-.01$, $p=.98$ ), and there was a negative correlation between time of day ratios and discrimination ratios during the final four test sessions $(r=-.531, p<.05)$ : Rats that were more adept at responding to the reinforced than the nonreinforced configurations were less likely to show different overall levels of responding in the morning and afternoon (see Fig. 4). The rates of activity during the corresponding periods in Experiment 2 were similar in the morning and afternoon when pooled across all sessions of training ( $\mathrm{AM}=21.2 \mathrm{rpm}$ and $\mathrm{PM}=22.5 \mathrm{rpm}, F(1,15)=1.28, p>.05)$, but these rates were higher in the afternoon $(26.2 \mathrm{rpm})$ than the morning $(21.3 \mathrm{rpm})$ in the final 3 blocks of training $(F(1,15)=10.85$, $p<.05)$. Again, however, time of day ratios for all sessions did not correlate with the corresponding configural discrimination ratios, that were not statistically different from chance as a
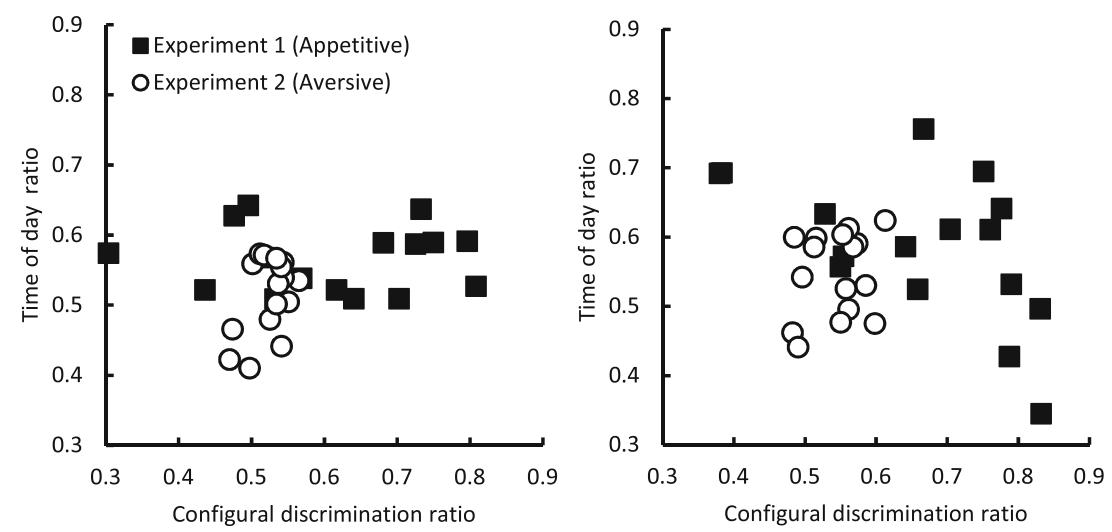

Fig. 4 Experiments 1 and 2: Relationship between time of day discrimination ratios and configural discrimination ratios over the entire course of training (left panel) and for the final sessions of training (right panel; see text for further details) 
group $(r=.45, p=.079)$; and there was no correlation between these two types of ratios for the final three blocks of training $(r=.22, p=.41$; see Fig. 4). These results provide little support for the idea that rats encoded time of day in terms of their relative deprivation state or level of arousal, at least insofar as our behavioral measures provided a sensitive assay of such processes.

Another possibility is that internal biological rhythms entrained to cues associated with the light cycle in the laboratory provided the basis for differentiating the morning and afternoon sessions. To evaluate this possibility, as opposed to accepting it by default, would require further experimental work to be conducted in which the light cycles were reversed or shifted between training and testing. For example, one could delay the onset of the vivarium lights by several hours so that rats are placed in the chambers at the same time as the afternoon session, but only one hour after the onset of the vivarium lights. If the critical (internal) cue for time of day is related to the light cycle in the vivarium, then this manipulation should result in behavior indicative of the morning sessions, but in the afternoon. However, the light cycle is only one of the potential zeitgebers in a laboratory (e.g., Aschoff, 1984).

To summarize The novel procedures described here provide automated measures of the acquisition of configural discriminations involving where and when appetitive and aversive events were presented. The results demonstrate that, as with other forms of simple conditioning, this learning takes the form of changes in conditioned responding (i.e., inactivity) that do not necessarily match the immediate unconditioned response to the unconditioned stimulus (i.e., hyperactivity; Experiment 2). Also, learning is not only evident as conditioned changes in behavior to the configural stimuli, but also the capacity of these stimuli to modulate unconditioned responding. These behavioural effects reveal important parallels between the acquisition of configural discriminations involving the components of episodic memory and simple conditioning.

Acknowledgments This research was supported by a grant from the Biotechnology and Biological Sciences Research Committee (UK); and was supported by a School of Psychology Studentship awarded to Natasha M. Dumigan.

\section{References}

Aschoff, J. (1984). Circadian timing. Annals of the New York Academy of Sciences, 423, 442-468.

Cain, S. W., Ko, C. H., Chalmers, J. A., \& Ralph, M. R. (2004). Time of day modulation of conditioned place preference in rats depends on the strain of rat used. Neurobiology of Learning and Memory, 81, 217-220.

Clayton, N. S., \& Dickinson, A. (1998). Episodic-like memory during cache recovery by scrub jays. Nature, 395, 272-274.

Fanselow, M. S. (1982). The postshock activity burst. Animal Learning and Behavior, 10, 448-454.

Fanselow, M. S. (1986). Associative vs. topographical accounts of the immediate shock freezing deficit in rats: Implications for the response selection rules governing species specific defensive reactions. Learning and Motivation, 17, 16-39.

Honey, R. C., \& Good, M. (2000). Associative modulation of the orienting response: Distinct effects revealed by hippocampal lesions. Journal of Experimental Psychology: Animal Behavior Processes, 26, 3-14.

Honey, R. C., \& Watt, A. (1999). Acquired relational equivalence between contexts and features. Journal of Experimental Psychology: Animal Behavior Processes, 25, 324-333.

Honey, R. C., Good, M., \& Manser, K. L. (1998a). Negative priming in associative learning: Evidence from a serial-habituation procedure. Journal of Experimental Psychology: Animal Behavior Processes, 24, 229-237.

Honey, R. C., Watt, A., \& Good, M. (1998b). Hippocampal lesions disrupt an associative mismatch process. Journal of Neuroscience, 18 , 2226-2230.

Honey, R. C., Iordanova, M. D., \& Good, M. (2014). Associative structures in animal learning: Dissociating elemental and configural processes. Neurobiology of Learning and Memory, 108, 96-103.

Iordanova, M. D., Good, M., \& Honey, R. C. (2008). Configural learning without reinforcement: Integrated memories for what, where and when. Quarterly Journal of Experimental Psychology, 61, 1785-1792.

Iordanova, M. D., Burnett, D., Aggleton, J. P., Good, M., \& Honey, R. C. (2009). The role of the hippocampus in mnemonic integration and retrieval: Complementary evidence from lesion and inactivation studies. European Journal of Neuroscience, 30, 2177-2189.

Iordanova, M. D., Burnett, D., Good, M., \& Honey, R. C. (2011a). Pattern memory involves both elemental and configural processes: Evidence from the effects of hippocampal lesions. Behavioral Neuroscience, 125, 567-577.

Iordanova, M. D., Good, M., \& Honey, R. C. (2011b). Retrieval-mediated learning involving episodes requires synaptic plasticity in the hippocampus. Journal of Neuroscience, 31, 7156-7162.

Lin, T. E., \& Honey, R. C. (2010). Analysis of the content of configural representations: The role of associatively evoked and trace memories. Journal of Experimental Psychology: Animal Behavior Processes, 36, 501-505.

Lin, T. E., Dumigan, N. M., Dwyer, D. M., Good, M. A., \& Honey, R. C. (2013). Assessing the encoding specificity of associations with sensory preconditioning procedures. Journal of Experimental Psychology: Animal Behavior Processes, 39, 67-75.

McDonald, R. J., Hong, N. S., Ray, C., \& Ralph, M. R. (2002). No time of day modulation or time stamp on multiple memory tasks in rats. Learning and Motivation, 33, 230-252.

Means, L. W., Arolfo, M. P., Ginn, S. R., Pence, J. D., \& Watson, N. P. (2000a). Rats more readily acquire a time-of-day go no-go discrimination than a time-of-day choice discrimination. Behavioural Processes, 52, 11-20.

Means, L. W., Ginn, S. R., Arolfo, M. P., \& Pence, J. D. (2000b). Breakfast in the nook and dinner in the dining room: time-of-day discrimination in rats. Behavioural Processes, 49, 21-33.

Preston, G. C., Dickinson, A., \& Mackintosh, N. J. (1986). Contextual conditional discriminations. Quarterly Journal of Experimental Psychology, 38B, 217-237. 
Thorpe, C. M., Bates, M. E., \& Wilkie, D. M. (2003). Rats have trouble associating all three parts of the time-place-event memory code. Behavioural Processes, 63, 95-110.
Wagner, A. R. (1981). SOP: A model of automatic memory processing in animal behavior. In N. E. Spear \& R. R. Miller (Eds.), Information processing in animals: Memory mechanisms (pp. 5-148). Hillsdale, NJ: Erlbaum. 